\title{
Method for Evaluating Stability of Highly Concentrated Emulsion and Its Application
}

\author{
LEI Qun ${ }^{1}$, ZHANG Yurong ${ }^{2}$, LUO Jianhui 1,3, HAN Rongcheng ${ }^{2}$, GENG Xiangfei ${ }^{1,3}$, \\ LÜ Xiaodong ${ }^{2}$, LIU Yan ${ }^{2}$, WANG Yuan ${ }^{2, *}$ \\ ${ }^{1}$ Research Institute of Petroleum Exploration and Development, Petrochina Company Limited, Beijing 100083, P. R. China. \\ ${ }^{2}$ Beijing National Laboratory for Molecular Science, State Key Laboratory for Structural Chemistry of Unstable and Stable Species, \\ College of Chemistry and Molecular Engineering, Peking University, Beijing 100871, P. R. China. \\ ${ }^{3}$ Key Laboratory of Nano Chemistry, China National Petroleum Corporation, Beijing 100083, P. R. China.
}

\begin{abstract}
The average diameter and size distribution of dispersed-phase droplets are important factors affecting the properties of emulsions, and the changes in these parameters with time and environment can be used to evaluate the emulsion stability. Traditional size characterization methods such as dynamic light scattering (DLS) are not applicable to highly concentrated emulsions. Herein, we report an imaging-based method to measure the droplet size in highly concentrated emulsions. This method comprises three steps: 1) emulsions are labeled with a
\end{abstract}

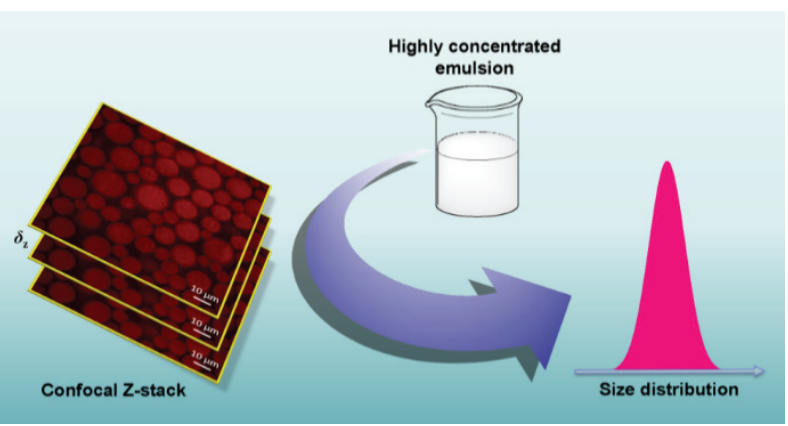
fluorescent dye, 2) three optical slices with a certain distance between two adjacent focal plans are measured sequentially via confocal laser scanning microscopy, 3) the sizes of dispersed-phase droplets are determined from the apparent diameters of droplets in the optical slices. When the apparent diameter of a droplet in the three optical slices increases or decreases monotonically, droplet diameter is calculated according to the following equations: $D_{\mathrm{c} 1-2}=\left\{D_{2}^{2}+\left[\left(D_{1}^{2}-D_{2}^{2}\right) / 4 \delta_{z}+\right.\right.$ $\left.\left.\delta_{z}\right]^{2}\right\}^{1 / 2}$ or $D_{\mathrm{C} 2-3}=\left\{D_{3}^{2}+\left[\left(D_{2}^{2}-D_{3}^{2}\right) / 4 \delta_{z}+\delta_{z}\right]^{2}\right\}^{1 / 2}$, where $D_{1}, D_{2}, D_{3}$ is the apparent diameter of the droplet measured from the consecutively-obtained optical slices $1-3$, respectively; $D_{\mathrm{C} 1-2}$ represents the calculated diameter of the droplet from the slices 1 and 2 , and $D_{\mathrm{C} 2-3}$ is that from the slices 2 and 3 , and $\delta_{z}$ is the distance between two focal planes of the adjacent optical slices. To avoid an obvious interference from the droplet movement, we use the equation $2 \mid D_{c 1-2}-$ $D_{\mathrm{c} 2-3} / /\left(D_{\mathrm{C} 1-2}+D_{\mathrm{c} 2-3}\right)=X$, where a smaller $X$ value indicates a less extent of movement during measurement, and that the calculated average diameter $\left(D_{\mathrm{c} 1-2}+D_{\mathrm{c} 2-3}\right) / 2$ is closer to the measured size of the droplet. The experimental results showed that when $X$ was $15 \%$, the difference between the calculated and measured diameters was about $10 \%$. When $X$ was less than $15 \%$, the calculated average droplet diameter was adopted as an effective diameter. However, when the condition $D_{1}=D_{2} \geq D_{3}$ (or $D_{3}=D_{2} \geq D_{1}$ ) was met, $D_{2}$ was used as the effective droplet diameter. The present method combines the advantages of fluorescent labeling, double optical slices analysis, and a strategy for eliminating the error caused by droplet movement. The stability of highly concentrated emulsions (oil volume percentage: $60 \%-80 \%$ ), prepared by mixing a crude oil model mixture containing $n$-decane, naphthaline, decalin, and tetraphenylporphyrin $(92.3 \%, 4.1 \%, 3.6 \%$, and $0.1 \%$ by mass, respectively) with aqueous solutions containing surfactants, was studied with the proposed method. The experimental results indicated that the present method allowed for the effective and accurate measurement of the anti-coalescence stability of emulsion dispersed-phase droplets. In contrast, the widely adopted "bottle test" method could not provide accurate information on the anti-coalescence stability of the dispersed phase droplets.

Received: February 11, 2018; Revised: March 7, 2018; Accepted: March 7, 2018; Published online: March 14, 2018.

${ }^{\star}$ Corresponding author. Email: wangy@pku.edu.cn; Tel.: +86-10-62757497.

The project was supported by the PetroChina Scientific Research and Technology Development Project (2014A-1001) and the National Key Research and Development Program of China (2016YFE0118700).

中国石油天然气股份有限公司科学研究与技术开发项目(2014A-1001)和国家重点研发计划项目(2016YFE0118700)资助

(C) Editorial office of Acta Physico-Chimica Sinica 
Key Words: Emulsion; Anti-coalescence stability; Salt-tolerance; Micro-imaging analysis; Double optical slice analysis

\title{
高浓度乳液稳定性评价方法及其应用
}

\author{
雷群 ${ }^{1}$, 张玉荣 ${ }^{2}$, 罗健辉 ${ }^{1,3}$, 韩荣成 ${ }^{2}$, 耿向飞 ${ }^{1,3}$, 吕晓东 ${ }^{2}$, 刘岩 ${ }^{2}$, 王远 ${ }^{2,}{ }^{*}$ \\ 1 中国石油天然气股份有限公司勘探开发研究院，北京 100083 \\ 2 北京大学化学与分子工程学院, 北京分子科学国家实验室, 分子动态与稳态结构国家重点实验室, 北京 100871 \\ 3 中国石油天然气集团公司纳米化学重点实验室, 北京 100083
}

\begin{abstract}
摘要: 分散相液滴的粒径及其分布是影响乳液性质的重要因素, 其随时间及环境的变化可用于评价乳液的稳定性。动态 光散射等方法难以用于准确测量高浓度乳液液滴粒径。本文报道了一种可用于准确、高效测量乳液液滴粒径的成像表征 方法。该方法采用荧光染料标记乳液液滴, 利用激光扫描共聚焦苂光显微成像技术获取三张焦平面间距确定的乳液光学 切片, 由光学切片给出的乳液液滴表观直径计算进而确定所测量乳液液滴的粒径。我们将上述方法用于表征高浓度原油 模拟物-水乳液的稳定性, 结果表明本文提出的方法可以准确、高效地测量乳液液滴的抗凝聚稳定性, 而目前广泛采用 的 “瓶试法” 则难以反映乳液液滴的抗凝聚稳定性。
\end{abstract}

关键词: 微乳; 抗凝聚稳定性; 耐盐稳定性; 显微成像分析; 双光学切片分析 中图分类号：0648

\section{1 引言}

乳液是一种液体以微小液滴的方式分散于另 一种不相溶的液体中形成的分散体系, 其中液滴 为内相, 连续相为外相 1 。乳液广泛应用于油田开 采、食品、医药、化工等诸多领域, 其稳定性是 乳液的重要性质之一 2,3 。目前评价乳液稳定性常 用的方法为 “瓶试法”, 即通过测量乳液中析出水 的体积与制备乳液时所用水的体积比随时间的变 化来评价乳液的稳定性 ${ }^{4-6}$ 。该方法因操作简单而 被广泛采用 ${ }^{7}$, 但它不能反映乳液微观结构的变 化, 在很多情况下难以区分导致乳液不稳定的因 素。乳液中分散相液滴的粒径及其分布是影响乳 液性质的重要因素, 其随时间及环境的变化可用 于评价乳液的稳定性。

迄今, 人们已发展了一些测量乳液分散相液滴 粒径方法, 例如, 动态光散射法(Dynamic Light Scattering, DLS) ${ }^{8-13}$ 、聚焦光反射测量方法(Focused Beam Reflectance Measurement, FBRM) ${ }^{14-18}$ 和光学 显微镜法 19,20 。然而这些方法难以满足油田开采等 领域对高浓度乳液中分散相液滴真实粒径及其分 布进行快速、准确分析的要求。DLS 并不能直接 测量乳液分散相液滴粒径, 而是通过其它物理量 的转换获得等效尺寸的信息, 当样品分散相浓度 较高时还需要对样品进行稀释, 其对高浓度乳液 的测量结果与真实情况有较大差别。FBRM 利用 激光焦点在分散相液滴上扫过的弦长计算液滴的
粒径, 该方法仅适用于测量粒径较大的分散相, 其测量结果受样品浓度等因素干扰严重 ${ }^{21,22}$ 。光学 显微镜法可以给出直观的乳液分散相液滴形貌特 征, 但该方法通常要求所测样品中分散相呈单层 分布, 否则在形成二维图像时将导致不同分散相 液滴的图像重合, 因此该方法也难以用于测量分 散相浓度较高的样品 ${ }^{22}$ 。

激光扫描共聚焦显微成像技术 (Confocal Laser Scanning Microscope，CLSM)采用逐点照明 和空间针孔调制去除来自非焦点处的散射光, 大 大提高了图像的对比度和空间分辨率, 已被广泛 用于活细胞的动态检测、生物样品光学切片和三 维结构重构等方面的研究 ${ }^{23-28}$ 。将 CLSM 应用于 乳液分散相液滴粒径分析时, 会遇到两个棘手的 问题: 首先, 难以保证所获得的某张图像的焦平 面对应的是液滴的最大截面; 其次, 在做光学切 片分析时, 样品因布朗运动可能发生随机的移动。 因而采用传统的共聚焦成像分析方法测量乳液分 散相液滴粒径及其分布不仅耗时长、效率低, 而 且还存在较大误差。

为解决上述问题, 本文提出一种准确、高效 的乳液分散相液滴粒径成像分析方法, 可用于测 量高浓度乳液液滴粒径或评价乳液的抗凝聚稳定 性。我们采用该方法研究了表面活性剂、原油模 拟物和水形成的分散体系的稳定性。 


\section{2 实验部分}

\section{1 实验试剂和仪器}

正癸烷、十六烷基三甲基溴化铵 $(\mathrm{CTAB})$ 、萗 和十氢荎(国药集团化学试剂有限公司), 聚乙二醇 辛基苯基醚(OP-10, 江苏海安石油化工厂), 四苯 基卟啉(TPP, 上海笛柏化学品技术有限公司)和氯 化钠(北京化工厂)试剂均为分析纯。实验用水采用 $18 \mathrm{M} \Omega \cdot \mathrm{cm}$ 超纯水。

采用上海嫩谷电机设备有限公司生产的 NSR-I 数显高速乳化均质机制备乳液; 采用转盘 式共聚焦荧光显微镜(Observer, Z1, ZEISS)拍摄 乳液分散相液滴的光学切片图像。

\section{2 原油模拟物-水乳液的制备}

原油模拟物制备: 将 $3.0 \mathrm{~g}$ 䒺和 $3.0 \mathrm{~mL}$ 十氢 䒬溶解于 $94.0 \mathrm{~mL}$ 正癸烷中, 再将 $10.1 \mathrm{mg}$ 苂光剂 四苯基卟啉(TPP)溶于上述溶液中制得原油模拟 物备用。

将 $6.0 \mathrm{~mL}$ 上述原油模拟物加入到 $4.0 \mathrm{~mL}$ 含乳 化剂 OP-10 的水溶液中(OP-10 在水溶液中的质量 百分比浓度为 $0.5 \%$ ), 在 $6000 \mathrm{r} \cdot \mathrm{min}^{-1}$ 转速下搅拌 $2 \mathrm{~min}$, 得到乳液 $\mathrm{I}$ 。

将 $8.0 \mathrm{~mL}$ 原油模拟物加入到 $2.0 \mathrm{~mL}$ 含乳化剂 OP-10 的水溶液中 $(\mathrm{OP}-10$ 在水溶液中的质量百分 比浓度为 $0.5 \%$ ), 按上述条件搅拌, 得到乳液 II。

将 $6.0 \mathrm{~mL}$ 原油模拟物加入到 $4.0 \mathrm{~mL}$ 含乳化剂 OP-10 的氯化钠水溶液中 $(\mathrm{OP}-10$ 在水溶液中的质 量百分比浓度为 $0.5 \%$, 氯化钠水溶液的质量百分 比浓度为 $20.0 \%$ ), 按上述条件搅拌, 得到乳液 III。

将 $6.0 \mathrm{~mL}$ 原油模拟物加入到 $4.0 \mathrm{~mL}$ 含乳化剂 CTAB 的水溶液中 $(\mathrm{CTAB}$ 在水溶液中的质量百分 比浓度为 $0.5 \%$ ), 按上述条件摚拌, 得到乳液 IV。

将 $6.0 \mathrm{~mL}$ 原油模拟物加入到 $4.0 \mathrm{~mL}$ 含乳化剂 $\mathrm{CTAB}$ 的氯化钠水溶液中 $(\mathrm{CTAB}$ 在水溶液中的质 量百分比浓度为 $0.5 \%$, 氯化钠水溶液的质量百分 比浓度为 $20.0 \%$ ), 按上述条件搅拌, 得到乳液 V。

\section{3 乳液成像分析方法}

将待测乳液置于共聚焦血中, 使用转盘式共 聚焦苂光显微镜(Observer, Z1, ZEISS)对待测样 品进行连续步进式光学切片荧光成像分析。苂光 显微成像条件: 物镜, Plan-Apochromat 63×/1.40 Oil M27; 观察方式, 荧光成像; 激发波长, $405 \mathrm{~nm}$; 检测波长, $(629 \pm 31) \mathrm{nm}$; 相邻两张光学切片的纵 向间距(焦平面间距) $\delta_{\mathrm{z}}$ 为 $0.5 \mu \mathrm{m}$ 。

\section{3 结果与讨论}

\section{1 高浓度乳液分散相液滴粒径测量方法}

如前所述，采用传统的共聚焦成像分析方法 测量乳液分散相液滴粒径, 存在耗时长且测量结 果不准确等问题。为解决上述问题, 我们发展了 一种准确、高效的乳液分散相液滴粒径的成像分 析方法, 即用荧光染料标记乳液, 采用激光扫描 共聚焦显微镜对所标记乳液以连续步进的方式进 行光学切片苂光成像分析, 获取三张焦平面间距 确定的乳液光学切片。测量上述三张光学切片图 像中液滴的表观直径, 当三张光学切片图像中某 液滴表观直径从第一张至第三张为连续递增或递 减时, 按公式(1)和(2)分别计算出同一液滴粒径的 计算值。

$$
\begin{aligned}
& D_{\mathrm{C} 1-2}=\left\{D_{2}^{2}+\left[\left(D_{1}^{2}-D_{2}^{2}\right) / 4 \delta_{\mathrm{z}}+\delta_{\mathrm{z}}\right]^{2}\right\}^{1 / 2} \\
& D_{\mathrm{C} 2-3}=\left\{D_{3}^{2}+\left[\left(D_{2}^{2}-D_{3}^{2}\right) / 4 \delta_{\mathrm{z}}+\delta_{\mathrm{z}}\right]^{2}\right\}^{1 / 2}
\end{aligned}
$$

公式(1)和(2)依据图 1 所示的几何关系得出, 其中, $D_{1} 、 D_{2} 、 D_{3}$ 分别为从第一张、第二张和第三张光 学切片图像中测得的同一液滴的表观直径; $D_{\mathrm{C} 1-2 、}$ $D_{\mathrm{C} 2-3}$ 分别为利用第一张与第二张光学切片图像和 利用第二张与第三张光学切片图像计算的液滴直 径; $\delta_{\mathrm{z}}$ 为相邻两张光学切片焦平面之间的间距(图 $1)$ 。

设 $2\left|D_{\mathrm{C} 1-2}-D_{\mathrm{C} 2-3}\right| /\left(D_{\mathrm{C} 1-2}+D_{\mathrm{C} 2-3}\right)=X, X$ 值 越小, 表明粒子在测量中移动的程度越小, 计算 值 $\left(D_{\mathrm{C} 1-2}+D_{\mathrm{C} 2-3}\right) / 2$ 越接近粒子的真实粒径。本文 作者的实验结果表明, 当 $X$ 小于 $15 \%$ 时, 所获得 的计算值与从数十张光学切片图像中寻找最大截 面的方法获得的实验结果相差不大(差值 $10 \%$ )。 因此, 本文中将 $X<15 \%$ 所对应的平均粒径值取为 所测液滴的有效粒径。

另一方面, 当三张光学切片图像中某液滴的 表观直径满足 $D_{1}=D_{2} \geq D_{3}$ 或 $D_{1} \leq D_{2}=D_{3}$ 时, 则 取相等的表观直径为所测液滴的有效粒径。

根据所测得液滴(200个以上)的有效粒径可获 得乳液液滴的平均粒径及粒径分布。该测定方法 结合了乳液苂光染料染色、双光学切片成像分析 和移动误差消除等策略, 可用于准确、高效地测

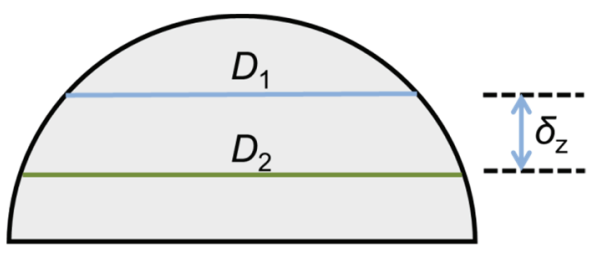

图 1 液滴直径测量与计算方法示意图

Fig. 1 Schematic diagram of the droplet diameter measurement and calculation method. 
定高浓度原油模拟物-水乳液液滴粒径。

\section{2 乳液液滴有效粒径测量方法验证}

如实验部分所述, 我们以 OP-10 作为乳化剂, 将原油模拟物(质量百分比: 正癸烷 $92.3 \%$ 、萗 4.1\% 和十氢荎 $3.6 \%$, TPP $0.1 \%$ ) 分散于水中, 制得油 相体积浓度为 $60.0 \%$ 的乳液 I。采用转盘式共聚焦 荧光显微镜(Observer, Z1, ZEISS)对该乳液进行 步进式成像分析, 连续拍摄了 30 张光学切片, 每 张曝光时间 $90 \mathrm{~ms}$, 焦平面间距 $0.5 \mu \mathrm{m}$ 。选择其中 任意相邻的三张光学切片图像 (图 $2 \mathrm{~A}$, 焦平面位置 $Z$ 为 6-8, 选择图像的原则为所测量液滴的图像清 晰), 按照前述(3.1)乳液分散相液滴有效粒径计算 方法获得了乳液 I 荧光图像中 1 至 3 号液滴的有 效粒径, 结果列于表 $1\left(D_{\mathrm{c}}\right)$ 。表 1 中 $D_{\max }$ 为从 30 张光学切片中找出的 1 至 3 号液滴的最大截面直 径, 各液滴最大截面直径对应的光学切片图像如 图 2B 所示。

对比图 $2 \mathrm{~A}$ 和 $2 \mathrm{~B}$ 可知, 焦平面位置对液滴的 表观直径有显著影响。因此, 共聚焦显微镜照片 上某液滴的表观粒径与其真实粒径之间可能存在 很大差别。以图 2 中的 1 号液滴为例, 在不同的 光学切片 (如 $Z=6$ 和 $Z=16$ ) 图像中的表观直径有 较大差别(16.9 和 $22.2 \mu \mathrm{m})$ 。因此, 若取单张光学 切片图像中各液滴的表观直径作为乳液液滴的粒 径, 会存在较大误差。本文提出根据三张光学切 片图像中给出的乳液液滴表观直径及其纵向间 距, 计算得出液滴的真实粒径。由该方法得到的 1 至 3 号液滴的有效粒径分别为 22.5、22.0 和 8.1 $\mu \mathrm{m}$ 。上述粒径值与从 30 张光学切片图像中寻找的 相应液滴的最大截面直径(22.2、20.9 和 $8.1 \mu \mathrm{m})$ 非

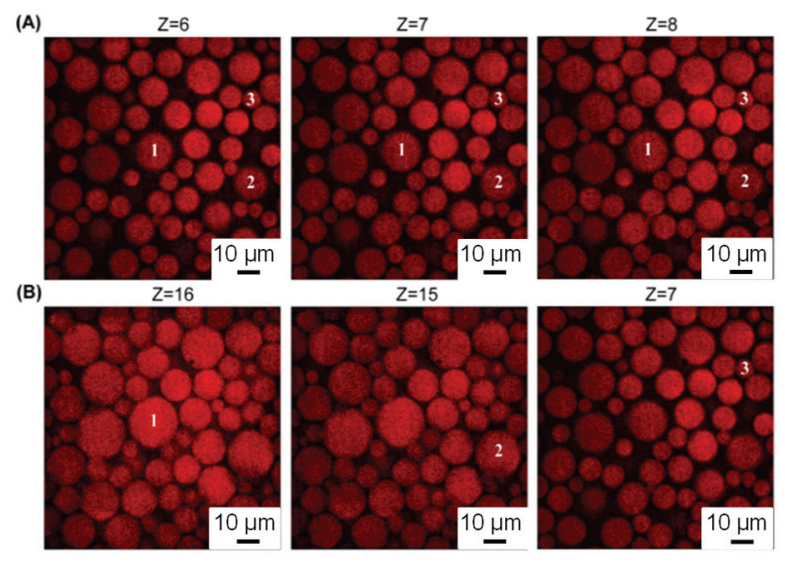

图 2 不同焦平面位置处乳液 $\mathbf{I}$ 的荧光显微图像

Fig. 2 Fluorescence images of the emulsion $I$ at different focal plane positions.

$Z$ represents the position of focal plane.
常接近, 表明本文发展的乳液液滴粒径测量法具 有较好的准确性。另外, 本文报道的方法仅需拍 摄乳液的 3 张光学切片, 即可实现对高浓度乳液 液滴粒径的准确测量, 相较于从数十张光学切片 中寻找最大截面的测量方法具有更高的效率, 且 可以减小乳液液滴随机运动导致的误差。

\section{3 高浓度原油模拟物-水乳液稳定性表征}

如实验部分所述, 我们以乳化剂 OP-10、所 配制原油模拟物、水制得了油相体积浓度为 $60.0 \%$ 的乳液 I 和油相体积浓度为 $80.0 \%$ 的乳液 II。首先 以传统的 “瓶试法” 来考察乳液的稳定性。乳液 I 和乳液 II 放置 $5 \mathrm{~h}$ 后, 油相体积浓度为 $60.0 \%$ 的乳 液 I 出现了分层现象, 而油相体积浓度为 $80.0 \%$ 的 乳液 II 却未出现分层现象。

我们采用本文所述成像方法及成像条件，分 别对新制的乳液 I 和乳液 II 进行荧光成像分析, 然后再对放置 $5 \mathrm{~h}$ 后再经缓慢摇匀的乳液 $\mathrm{I}$ 和乳液 II 进行荧光成像分析, 两次所拍摄的苂光成像图 如图 3 所示。

按照本文所述乳液分散相液滴有效粒径测量 方法, 测得乳液 I 和乳液 II 的粒径分布如图 4 所 示。由图 4 可见, 实际测得乳液 I 和乳液 II 液滴 的平均粒径及其分布很接近且在观测时间内未发 生明显变化。这表明乳液 I 和乳液 II 在放置 $5 \mathrm{~h}$ 后, 其液滴之间并没有发生凝聚现象, 即乳液 I 和乳液 II 均具有较好的抗凝聚稳定性。因此, “瓶 试法” 之所以反映出乳液 II 的稳定性高于乳液 I, 是由于当油水比为 $60.0 \%$ 时, 在新制备的乳液中, 分散相液滴均匀地分散在水相中，随着放置时间 的延长, 乳液液滴在浮力作用下上浮, 呈较为紧 密的堆积状态, 而多余的水(含有少量乳液液滴) 则留在了下层, 使乳液 I 出现了分层现象。而当 油水比为 $80.0 \%$ 时, 分散相形成更多紧密堆积的 乳液液滴, 填满这些乳液液滴之间空隙所需的水 量增加, 而制备这种乳液时所加的水全部填充到 乳液液滴之间的空隙中了, 因此乳液 II 未出现分

表 1 乳液分散相液滴粒径分析结果

Table 1 The sizes of droplets in different emulsion

\begin{tabular}{ccccccc}
\multicolumn{7}{c}{ samples. } \\
\hline Droplet & $D_{1} / \mu \mathrm{m}$ & $D_{2} / \mu \mathrm{m}$ & $D_{3} / \mu \mathrm{m}$ & $D_{\mathrm{C}} / \mu \mathrm{m}$ & $D_{\max } / \mu \mathrm{m}$ & $Y / \%$ \\
\hline 1 & 16.9 & 17.7 & 18.4 & 22.5 & 22.2 & 1.3 \\
2 & 14.4 & 15.3 & 16.4 & 22.0 & 20.9 & 5.0 \\
3 & 8.1 & 8.1 & 7.9 & 8.1 & 8.1 & 0 \\
\hline
\end{tabular}

$D_{1}, D_{2}, D_{3}$ are the apparent diameters of droplets in different optical slice

images, $D_{\mathrm{C}}$ is the calculated particle size of droplet and $D_{\max }$ is the

maximum cross-section diameter of droplet, $Y=\left(D_{\mathrm{C}}-D_{\max }\right) / D_{\mathrm{C}} \times 100 \%$. 
Oh
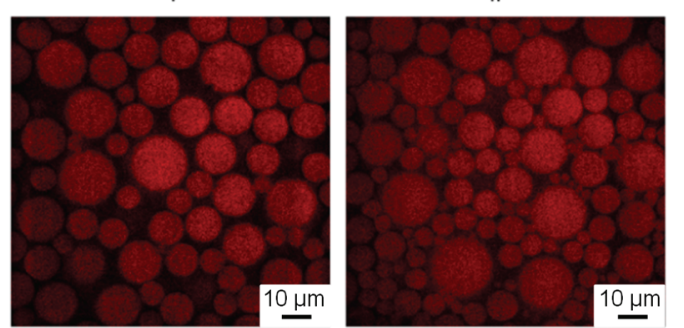

$5 \mathrm{~h}$
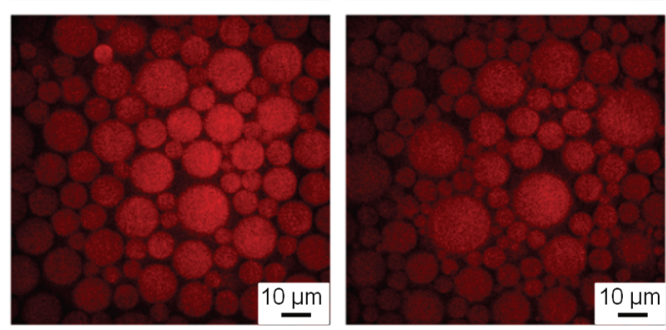

图 3 新制备及放置 $5 \mathrm{~h}$ 后乳液 I 和乳液 II 的 荧光显微图像

Fig. 3 Fluorescence images of emulsions I and II 0 and $5 \mathrm{~h}$ after the preparation.

Oh
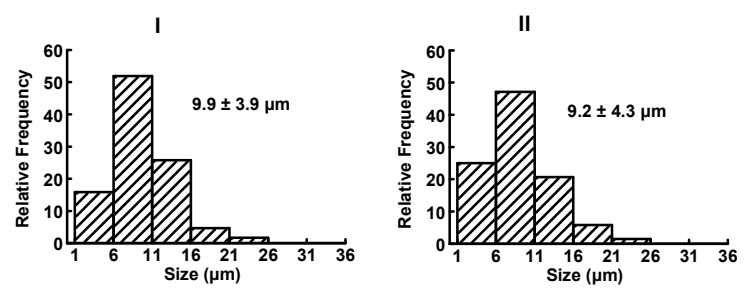

$5 \mathrm{~h}$
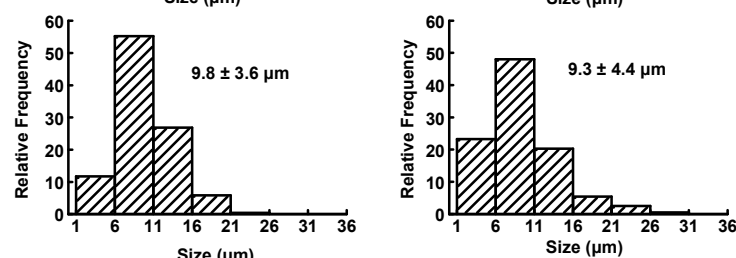

图 4 乳液 $I$ 和乳液 II 分散相液滴粒径分布图

Fig. 4 Size distributions of dispersed-phase droplets in emulsion I and emulsion II.

层现象。

上述实验结果表明，用传统的 “瓶试法” 评 价乳液的稳定性具有一定的局限性, 乳液的动力 学不稳定、乳液液滴絮凝或融合都可能造成乳液 分层, 传统的 “瓶试法” 并不能很好地反映乳液 分层的原因。此外, 对于高浓度的乳液, 即使其 液滴紧密地堆积在一起, 由于分散介质体积小, 乳液也可能不出现分层现象。而本文提出的评价 方法弥补了 “瓶试法” 的不足, 可以更准确地评 价乳液液滴的抗凝聚稳定性。

\section{4 不同乳化剂形成乳液的耐盐稳定性}

我们以乳化剂 OP-10、原油模拟物、水制得了 油相体积浓度为 $60.0 \%$ 的乳液 I; 由乳化剂 OP-10、 原油模拟物、 $20.0 \%$ 氯化钠水溶液制得油相体积浓

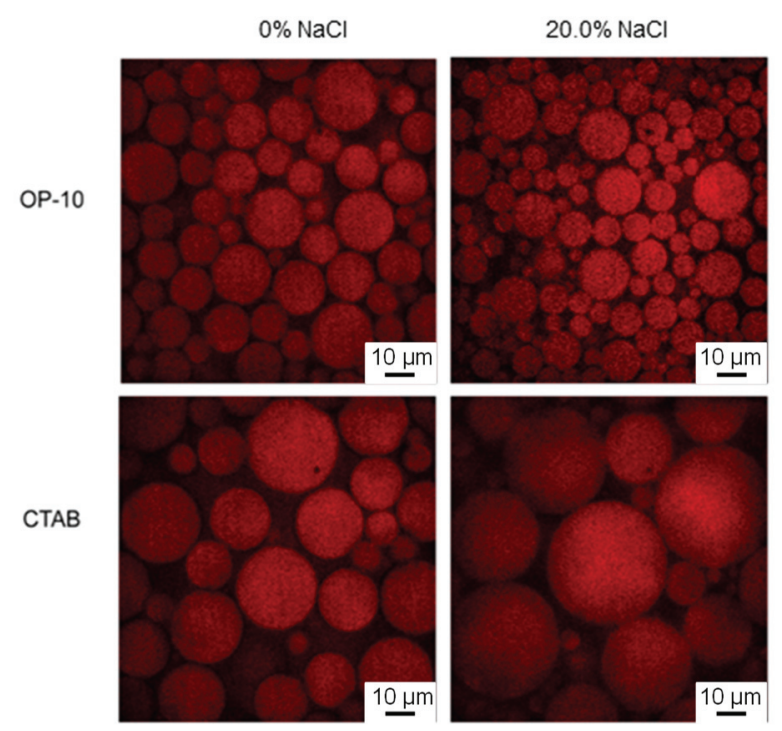

图 5 采用乳化剂 OP-10 和 CTAB 及不同质量浓度的 氯化钠溶液制得乳液的荧光图像

Fig. 5 Fluorescence images of emulsions prepared with OP-10, CTAB and aqueous solutions with different contents of sodium chloride.
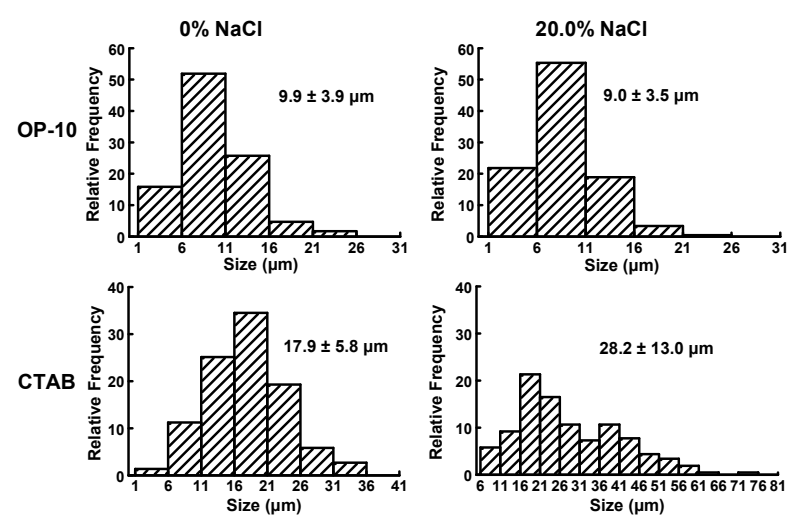

图 6 采用乳化剂 OP-10 和 CTAB 及不同质量浓度的 氯化钠溶液制得乳液液滴粒径分布图

Fig. 6 Size distributions of emulsions prepared with

OP-10, CTAB and aqueous solutions with different contents of sodium chloride.

度为 $60.0 \%$ 的乳液 III。采用上述成像方法及成像 条件对乳液 I 和乳液 III 进行了苂光成像分析。其 苂光图像如图 5 所示。按照本文提出的乳液分散 相液滴有效粒径测量方法，测得乳液 I 和乳液 III 分散相液滴粒径及分布如图 6 所示。由图 6 可见， 乳液 I 和乳液 III 分散相液滴的平均粒径及粒径分 布变化不大, 这表明 OP-10 乳化剂形成的乳液具 有较好的耐盐稳定性。OP-10 属于非离子型表面 活性剂, 乳液液滴之间的空间位阻等作用是阻止 其液滴之间发生絮凝 (flocculation) 或融合 (coalescence)的主要因素。因此, OP-10 乳化剂形 
成的乳液受强电解质的影响较小, 表现出很好的 耐盐稳定性。

如实验部分所述, 我们以乳化剂 CTAB, 原油 模拟物, 水制得了油相体积浓度为 $60.0 \%$ 的乳液 IV; 由乳化剂 CTAB, 原油模拟物, $20.0 \%$ 氯化钠 水溶液制得油相体积浓度为 $60.0 \%$ 的乳液 $\mathrm{V}$ 。采用 上述成像方法及成像条件对乳液 IV 和乳液 $V$ 进行 了荧光成像分析, 其荧光图像如图 5 所示。按照 上述乳液分散相液滴有效粒径测量方法, 测得乳 液 IV 和乳液 $\mathrm{V}$ 分散相液滴粒径尺寸及分布如图 6 所示。由图 6 可见, 与乳液 IV 相比, 乳液 V 分散 相液滴的粒径大且分布宽, 表明 CTAB 乳化剂形 成的乳液耐盐稳定性不高。CTAB 是阳离子表面活 性剂, 在 $20.0 \%$ 氯化钠水溶液环境中, 强电解质 的存在会使 CTAB 乳化剂形成的乳液液滴表面上 的双电层受到压缩, 降低了相邻液滴间的斥力, 液滴之间更易靠近, 进而发生液滴融合, 使液滴 粒径变大。

\section{4 结论}

本文报道了一种测定乳液分散相液滴粒径的 成像分析方法, 该方法结合了乳液苂光标记、双 光学切片成像分析和移动误差消除等策略, 可以 对浓度高、颜色深的乳液分散相液滴粒径及其分 布进行准确、高效的测定。

采用本文提出的方法研究了乳液I和II的抗凝 聚稳定性, 结果表明, 虽然乳液I和II经放置后分 层情况完全不同, 但它们液滴的抗凝聚稳定性相 似。我们考察了乳化剂OP-10和CTAB形成乳液的 耐盐稳定性, 结果表明当氯化钠水溶液浓度为 $20.0 \%$ 时, 乳化剂OP-10形成的乳液耐盐稳定性明 显优于CTAB形成的乳液的耐盐稳定性。本文提出 的方法克服了 “瓶试法” 的不足, 为深入研究高浓 度乳液微观结构的衍化提供了一种有效的方法。

\section{References}

(1) Wong, S. F.; Lim, J. S.; Dol, S. S. J. Pet. Sci. Eng. 2015, 135, 498. doi: 10.1016/j.petrol.2015.10.006

(2) Tadros, T. F. Applied Surfactants: Principles and Applications; Wiley-VCH: Weinheim, 2005; pp. 139-140.

(3) Zhang, X. G.; Liu, J. X.; Wang, H. Y.; Wang, M. Y.; Fan, Z. J. Acta Phys. -Chim. Sin. 2010, 26 (3), 617. [张晓光, 刘洁翔, 王海英, 王满意, 范志金. 物理化学学报, 2010, 26(3), 617.] doi: 10.3866/PKU.WHXB20100313

(4) Martínez-Palou, R.; Cerón-Camacho, R.; Chávez, B.; Vallejo, A. A.; Villanueva-Negrete, D.; Castellanos, J.; Karamath, J.; Reyes, J.;
Aburto, J. Fuel 2013, 113, 407. doi: 10.1016/j.fuel.2013.05.094

(5) da Fraga, A. K.; Oliveira, P. F.; Oliveira, L. F. S.; Magalhães, J.; Mansur, C. R. E. J. Appl. Polym. Sci. 2016, 133 (44), 44174 doi: 10.1002/app.44174

(6) Jing, J. Q.; Sun, J.; Zhou, J.; Shen, X. Y.; Tan, J. T.; Li, X. M.; Zhang, L. P. J. Dispersion Sci. Technol. 2016, 37 (7), 980. doi: 10.1080/01932691.2015.1077454

(7) Santos, D.; da Rocha, E. C. L.; Santos, R. L. M.; Cancelas, A. J.; Franceschi, E.; Santos, A. F.; Fortuny, M.; Dariva, C. Sep. Purif. Technol. 2017, 189, 347. doi: 10.1016/j.seppur.2017.08.028

(8) Less, S.; Vilagines, R. Fuel 2013, 109, 542. doi: 10.1016/j.fuel.2013.03.048

(9) Koppel, D. E. J. Chem. Phys. 1972, 57, 4814. doi: 10.1063/1.1678153

(10) Zhang, L. F.; Ying, H.; Yan, S.; Zhan, N. N.; Guo, Y. S.; Fang, W. J. Fuel 2018, 211, 197. doi: 10.1016/j.fuel.2017.09.066

(11) Xiang, N.; Lyu, Y.; Naesimhan, G. Food Hydrocolloids 2016, 52, 678. doi: 10.1016/j.foodhyd.2015.08.015

(12) Philips, L. A.; Ruffner, D. B.; Cheong, F. C.; Blusewicz, J. M.; Kasimbeg, P.; Waisi, B.; McCutcheon, J. R.; Grier, D. G. Water Res. 2017, 122, 431. doi: 10.1016/j.watres. 2017.06.006

(13) Chen, F.; Chen, Z. G.; Sun, H. Z.; Meng, F. X.; Ma, X. Y. Acta Phys.-Chim. Sin. 2016, 32 (3), 763. [陈芳, 陈助国, 孙会昭, 孟繁想, 马晓燕. 物理化学学报, 2016, 32 (3), 763.] doi: 10.3866/PKU.WHXB201512111

(14) Boxall, J. A.; Koh, C. A.; Sloan, E. D.; Sum, A. K.; Wu, D. T. Ind. Eng. Chem. Res. 2010, 49 (3), 1412. doi: 10.1021/ie901228e

(15) Greaves, D.; Boxall, J.; Mulligan, J.; Montesi, A.; Creek, J.; Sloan, E. D.; Koh, C. A. Chem. Eng. Sci. 2008, 63 (22), 5410. doi: 10.1016/j.ces.2008.07.023

(16) Muhaimin; Bodmeier, R. Polym. Int. 2017, 66 (11), 1448. doi: $10.1002 /$ pi.5436

(17) Hermanto, M. W.; Chow, P. S.; Tan, R. B. H. Cryst. Growth Des. 2010, 10, 3668. doi: $10.1021 / \operatorname{cg} 100533$ n

(18) Dave, K.; Luner, P. E.; Forness, C.; Baker, D.; Jankovsky, C.; Chen, S. AAPS PharmSciTech 2017, 19 (1), 155. doi: 10.1208/s12249-017-0819-9

(19) Katepalli, H.; Bose, A.; Hatton, T. A.; Blankschtein, D. Langmuir 2016, 32, 10694. doi: 10.1021/acs.langmuir. 6 b03289

(20) Anjali, T. G.; Basavaraj, M. G. Phys. Chem. Chem. Phys. 2017, 19, 30790. doi: 10.1039/C7CP04665K

(21) Ruf, A.; Worlitschek, J.; Mazzotti, M. Part. Part. Syst. Charact. 2000, 17, 167. doi: 10.1002/1521-4117(200012)17:4<167::AID-PPSC167> 3.0.CO;2-T

(22) Abidin, M. I. I. Z.; Raman, A. A. A.; Nor, M. I. M. Ind. Eng. Chem. Res. 2013, 52, 16085. doi: 10.1021/ie401548z

(23) Amos, W. B.; White, J. G. Biol. Cell 2003, 95, 335. 
doi: 10.1016/S0248-4900(03)00078-9

(24) Mun, S.; Kim, J.; McClements, D. J.; Kim, Y. R.; Choi, Y. Food Chem. 2017, 219, 297. doi: 10.1016/j.foodchem.2016. 09.158

(25) Iwai, M.; Yokono, M.; Kurokawa, K.; Ichihara, A.; Nakano, A. Sci. Rep. 2016, 6, 29940. doi: 10.1038/srep29940
(26) Villaseñor, R.; Collin, L. J. Vis. Exp. 2017, 129, 1. doi: $10.3791 / 56407$

(27) Paddock, S. W. Biotechniques 1999, 27, 992.

(28) Carlsson, K.; Danielsson, P. E.; Liljeborg, A.; Majlöf, L.; Lenz, R.; Åslund, N. Opt. Lett. 1985, 10, 53. doi: 10.1364/OL.10.000053 